\title{
Rapid Monolayer Neural Induction of induced Pluripotent Stem Cells Yields Stably Proliferating Neural Stem Cells
}

\author{
Katharina Gunther ${ }^{1 \#}$, Antje Appelt-Menzel ${ }^{2,3 \#}$, Chee Keong Kwok ${ }^{1}$, Heike Walles ${ }^{2,3}$, Marco Metzger ${ }^{2,3}$ and Frank Edenhofer ${ }^{1,4 *}$ \\ ${ }^{1}$ Institute of Anatomy and Cell Biology, Stem Cell and Regenerative Medicine Group, 97070 Wurzburg, Germany \\ ${ }^{2}$ University Hospital Würzburg, Chair Tissue Engineering and Regenerative Medicine, 97070 Wurzburg, Germany \\ ${ }^{3}$ Translational Center Würzburg "Regenerative Therapies for Oncology and Musculoskeletal Diseases", Branch of Fraunhofer Institute for Interfacial Engineering and \\ Biotechnology IGB, 97070 Würzburg, Germany \\ ${ }^{4}$ Institute of Molecular Biology, Department Genomics, Stem Cell Biology and Regenerative Medicine, Leopold-Franzens-University Innsbruck, 6020 Innsbruck, Austria \\ "These two authors have contributed equally to this work and are sharing the first authorship
}

\begin{abstract}
Objective: The induction of neural stem cells (NSCs) from human induced pluripotent stem cells (hiPSCs) developed into an important strategy to derive patient-specific neuronal and glial cells. Several neural differentiation protocols have been developed mainly involving laborious experimentation such as embryoid body (EB) formation or manual neural rosette isolation. The aim of this study is to develop a rapid neural induction protocol, which combines a previously published monolayer approach with common cultivation methods.
\end{abstract}

Methods and results: hiPSCs were differentiated into primitive NSCs (pNSC) using a rapid monolayer differentiation protocol within 7 days. pNSCs were expanded up to 5 passages and showed a downregulation of the pluripotency gene POU5F1 and expressed NSC markers such as SOX1, SOX2, Nestin and PAX6. In a second step we adapted pNSCs to a widely used FGF/EGF-dependent NSC state by culturing in media supplemented with FGF, EGF and Wnt agonist CHIR99021. Under these conditions, cells underwent a rapid and prominent morphological change to rosette-like structures. These cells remained proliferative for more than 30 passages and maintained the expression profile of neural marker genes. Moreover, they could be efficiently differentiated into neurons as well as GFAP- and S100ß-positive astrocytes.

Conclusion: We report a robust two-step neural induction protocol for the generation of hiPSC-derived NPCs, closing the gap between previously published monolayer protocols and commonly used FGF/EGF-containing media conditions. Our protocol will serve as a fast and efficient neural induction strategy to derive patient-specific neural cells for biomedical applications such as disease modeling and cell replacement therapy.

Keywords: Neural stem cells; induced pluripotent stem cells; neural induction; differentiation, monolayer; neurons; astrocytes

\section{Introduction}

The use of cellular reprogramming of human somatic cells into self-renewable human induced Pluripotent Stem Cells (hiPSCs) [1] represents a major breakthrough in biomedical research. hiPSCs can be differentiated into stably proliferating Neural Stem Cells (NSCs) and thus provide a heretofore unattainable, virtually unlimited access to patient-specific neural cells in a sustainable and standardized manner. Through this approach, hiPSC-type reprogramming and subsequent neural differentiation has enabled major progress in modeling of various neurological [2], neurodevelopmental [3] and psychiatric diseases [4] as well as complex physiological systems such as the bloodbrain barrier [5].

Commonly used neural differentiation protocols involve relatively time consuming and laborious differentiation methods passing the Embryoid Body (EB) stage to achieve induction of rosette-like structures, representing in vitro correlates of the neural tube. Manually picked rosettes are further stably maintained in media containing human basic Fibroblast Growth Factor (bFGF) and Epidermal Growth Factor (EGF) [6-10]. Such NSC lines allow robust generation of neuronal subtypes whereas other protocols have been reported to be more suitable for obtaining glial cells such as oligodendrocytes [11-13]. Recently, a rapid monolayer differentiation protocol has been published that allows neural induction within a few days, circumventing the $\mathrm{EB}$ formation stage [14]. However, the composition of the commercially available media is not fully declared. Furthermore, it remains elusive how the generated NSCs functionally compare to widely established FGF/EGF-dependent NSCs that passed an EB stage. This study aims to bridge this gap by applying the rapid monolayer induction protocol to hiPSCs with a subsequent shift to well-established defined media conditions, thereby generating a monolayer-derived Neural Precursor Cell (NPC) population, which depends on bFGF and EGF. We show that such rapidly and efficiently generated NPCs can be expanded, cryopreserved and employed for standardized differentiation into glial and neuronal lineages. Our protocol provides a robust basis to rapidly generate patient-specific neural cells for pharmacological studies, cell replacement therapies and tissue engineering.

\section{Materials and Methods}

\section{Culturing of hiPSCs}

hiPSCs cultivation was performed in feeder-free conditions using

*Corresponding author: Frank Edenhofer, Stem Cell and Regenerative Medicine Group, Institute of Anatomy and Cell Biology, University of Würzburg, Koellikerstrasse 6, 97070 Würzburg, Germany; and Department Genomics, Stem Cell Biology and Regenerative Medicine, Institute of Molecular Biology, Leopold-Franzens-University Innsbruck, Technikerstrasse 25, 6020 Innsbruck, Austria, Tel:+49 931 31-88113; +43 507 51411; Fax: +49 931 31-82087; E-mail: frank.edenhofer@uni-wuerzburg.de; Frank.Edenhofer@uibk.ac.at

Received June 03, 2016; Accepted June 14, 2016; Published June 20, 2016

Citation: Gunther K, Appelt-Menzel A, Kwok CK, Walles H, Metzger M, et al. (2016) Rapid Monolayer Neural Induction of induced Pluripotent Stem Cells Yields Stably Proliferating Neural Stem Cells. J Stem Cell Res Ther 6: 341. doi: 10.4172/21577633.1000341

Copyright: (c) 2016 Gunther K, et al. This is an open-access article distributed under the terms of the Creative Commons Attribution License, which permits unrestricted use, distribution, and reproduction in any medium, provided the original author and source are credited. 
Matrigel $^{\circ}$ (Corning) tissue culture dishes and daily change of $\mathrm{mTeSR}^{\mathrm{TM}} 1$ medium (Stem Cell Technologies). Passaging of cells was done at a maximum of $80 \%$ confluency or every $4-5$ days by using StemPro Accutase (Gibco) for $3-5 \mathrm{~min}$ at $37^{\circ} \mathrm{C}$, harvesting cells by the addition of DMEM/F-12 (Life Technologies) and centrifuging at $300 \times \mathrm{g}$ for 3 $\min$ at $4^{\circ} \mathrm{C}$. Cells were seeded in a ratio of 1:6 on freshly coated tissue culture plates as a single cell suspension and $10 \mu \mathrm{M}$ ROCK inhibitor StemMACS ${ }^{\text {TM }}$ Y27632 (Miltenyi Biotec) was added.

\section{Neural induction and adaptation to defined media conditions}

Primitive NSCs (pNSCS) differentiation from hiPSC lines AR1034ZIMA hiPSC clone 1 (ARIPS) [15] and IMR90 hiPSC clone 4 (IMR90-4, WiCell) [16] was performed as previously described by Yan et al. with slight modifications [14]. Briefly, hiPSCs were seeded at a density of $2-2.5 \times 10^{4}$ cells per $\mathrm{cm}^{2}$ on Matrigel ${ }^{\circledR}$ coated 6 -well tissue culture plates. After approximately 24 hours (day 0 of neural induction), reaching $15-20 \%$ confluence, medium was changed to PSC Neural Induction Medium (Life Technologies). Medium exchange was performed every other day until day 4 of neural induction. From day 4 to day 7, medium was changed daily due to higher confluency. pNSCs were passaged enzymatically using Accutase ${ }^{\circledR}$ at day 7 of neural induction. Cells were seeded in a density of $0.5-1 \times 10^{5}$ cells per $\mathrm{cm}^{2}$ on Matrigel ${ }^{\mathbb{B}}$ coated 6-well plates and cultured for 5 days in NSC expansion medium (49\% Neurobasal ${ }^{\mathbb{}}$ medium $+49 \%$ Advanced DMEM/F-12 (Life Technologies) + 2\% Neural Induction Supplement). After seeding, pNSCs were treated with $10 \mu \mathrm{M}$ ROCK inhibitor Stem MACS $^{\text {TM }}$ Y27632 (Miltenyi Biotec) overnight to prevent cell death. Neural expansion medium was changed every other day until day 5. To achieve a later stage rosette-like NSC-population, cells in passage 5 were adapted to FGF/EGF conditions, hereafter referred to as FGF/ EGF-NPCs, as described in the following. Cells were cultured in NPC medium composed of DMEM/F-12 + GlutaMAX ${ }^{\mathrm{TM}}-\mathrm{I}+0.8 \mu \mathrm{M}$ CHIR99021 (BioMol) + 1\% D-glucose enriched DMEM/F-12 (160 $\mathrm{mg} / \mathrm{mL}$ D-glucose (Sigma-Aldrich) added to DMEM/F-12)+10 ng/ $\mathrm{mL}$ human bFGF (PeproTech) $+10 \mathrm{ng} / \mathrm{mL}$ EGF (PeproTech) $+0.1 \%$ B27 Supplement (Life Technologies) + 1\% N2 Supplement (Life Technologies) [8] on $15 \mu \mathrm{g} / \mathrm{mL}$ Poly-L-ornithine (Sigma-Aldrich) $+1 \mu \mathrm{g} / \mathrm{mL}$ laminin (Sigma-Aldrich) coated 6-well plates. Coatings were diluted in phosphate-buffered saline (PBS; Sigma-Aldrich) and incubated separately for at least 2 hours at $37^{\circ} \mathrm{C}$. Poly-L-ornithine was washed 3 times before proceeding with laminin coating. Stably proliferating FGF/EGF-NPCs were split every 2-3 days at a ratio of 1:3. Cells were cryopreserved in $65 \%$ KnockOut Serum Replacement $($ Gibco $)+25 \%$ culturing medium $+10 \%$ dimethyl sulfoxide (DMSO, Carl Roth $\mathrm{GmbH}$ ).

\section{Directed differentiation of pNSCs and FGF/EGF-NPCs}

For neuronal differentiation pNSCs and FGF/EGF-NPCs were plated on Matrigel-coated coverslips at a density of $0.8-1.3 \times 10^{4}$ per $\mathrm{cm}^{2}$. One day after seeding the medium was changed to neuronal differentiation medium containing 49\% DMEM/F12 + 49\% Neurobasal medium $+1 \times \mathrm{N} 2$ Supplement $+1 \times$ B27 Supplement + $2 \mathrm{mM} \mathrm{L}$-Glutamine $+20 \mathrm{ng} / \mathrm{mL}$ brain derived neurotrophic factor (BDNF, Peprotech) $+20 \mathrm{ng} / \mathrm{mL}$ glial derived neurotrophic factor (GDNF, Peprotech) $+300 \mathrm{ng} / \mathrm{mL}$ cyclic adenosine monophosphate (cAMP, Sigma-Aldrich) and $200 \mu \mathrm{M}$ L-ascorbic acid (Sigma-Aldrich). Cells were differentiated at least 2-6 weeks while medium change was done every 2 - 3 days. Differentiation into astrocytes was adapted from the protocol of Reinhardt and colleagues [10]. Briefly, neural precursors were cultured for 2 days on Matrigel-coated plates in a medium containing 50\% DMEM/F-12 and 50\% Neurobasal medium supplemented with $10 \mathrm{ng} / \mathrm{mL}$ human bFGF, $10 \mathrm{ng} / \mathrm{mL}$ EGF, 1:200 N2 Supplement, 1:100 B27 Supplement without vitamin A, 1\% Penicillin/ Streptomycin and $2 \mathrm{mM} \mathrm{L-Glutamine.} \mathrm{Following} \mathrm{that,} \mathrm{culture} \mathrm{medium}$ was switched to N2 medium (DMEM/F-12 + GlutaMAX ${ }^{\mathrm{TM}}-\mathrm{I}+1 \% \mathrm{~N} 2$ Supplement $+1 \%$ Penicillin/Streptomycin) with $4 \%$ fetal calf serum (FCS, Bio\&Sell) and $10 \mathrm{ng} / \mathrm{mL}$ ciliary neurotrophic factor (CNTF, R\&D Systems) for at least 2 weeks. At confluency, dissociation with Accutase in a ratio of 1:3 was performed. After 2 weeks, conditions were changed to $\mathrm{N} 2$ medium $+4 \%$ FCS $+500 \mu \mathrm{M}$ dbcAMP (SigmaAldrich) for 7 days. Thereafter, cells were maintained in N2 medium + $4 \%$ FCS for at least 7 additional days, replacing the media every other day.

\section{Immunocytochemistry}

Cells were fixed with 4\% PFA (Applichem) solution in PBS for 15 minutes at room temperature and washed three times, 5 minutes each using PBS without $\mathrm{Ca}$ and $\mathrm{Mg}$. For blocking and permeabilizing, cells were incubated with $5 \%$ FCS $+0.1 \%$ Triton-X (Sigma-Aldrich) in PBS for one hour at room temperature. Next, incubation with respective antibody (Table S1) diluted in blocking solution was performed at $4^{\circ} \mathrm{C}$ over night. Cells were washed carefully three times using PBS and incubated with species-specific conjugated secondary antibodies diluted in PBS for one hour at room temperature protected from light. Having rinsed three times with PBS, 1:5000 DAPI (4',6-diamidino-2phenylindole) solution was applied for 15 minutes at room temperature in order to counterstain cell nuclei. Before coverslips were mounted with Mowiol'4-88 (Sigma-Aldrich) + DABCO $^{\circ}$ (Carl Roth) on glass slides, three further washing steps were carried out.

\section{Flow cytometry}

For each sample 0.5 to $1 \times 10^{6}$ cells were harvested. After excluding dead cells from the analysis using BD Horizon Fixable Viability Stain 450 (BD Biosciences) cell pellets were resuspended in staining buffer consisting of 5\% FCS in PBS. Having blocked unspecific binding sites, incubation for $10 \mathrm{~min}$ on ice was carried out and cells were pelleted by centrifugation $\left(300 \times \mathrm{g}, 5 \mathrm{~min}, 4^{\circ} \mathrm{C}\right)$. Cells were stained for $10 \mathrm{~min}$ at $4^{\circ} \mathrm{C}$ with TRA-1-60-PE (Miltenyi Biotec) antibody diluted in staining buffer. Next, centrifugation and washing with PBS was carried out before cells were carefully resuspended in PBS for analysis. To assure the lack of nonspecific binding and background fluorescence isotype and unstained samples were included as controls. Data was acquired using BD FACSCanto ${ }^{\mathrm{mm}}$ II (BD Biosciences) flow cytometer, capturing 50,000 events per sample. For analysis FlowJo 10.0.7 (Tree Star Inc) software was used.

\section{Quantitative Real-Time -PCR (qRT-PCR)}

For RNA isolation $2.5-5 \times 10^{5}$ cells were harvested, centrifuged and resuspended in $350 \mu \mathrm{L}$ RLT buffer $+1 \% \beta$-Mercaptoethanol. RNeasy Micro Kit (QIAGEN) was used following the instructions of the manufacturer. RNA concentration was determined by microplate reader Infinite M200 (TECAN). Up to $1 \mu \mathrm{g}$ RNA was transcribed into cDNA applying iScript ${ }^{\mathrm{TM}}$ cDNA Synthesis Kit (BIO-RAD) in a Thermocycler 48 (SensoQuest GmbH). qRT-PCR was performed with $1 \mu \mathrm{L}$ cDNA dilution and the SsoFast ${ }^{\mathrm{TM}}$ EvaGreen Supermix (BIORAD). Primers (for sequences see Table S2) were applied in a working concentration of $4 \mathrm{pmol} / \mu \mathrm{L}$. PCR was performed in the thermocycler SensQuest (BIO-RAD), with a 2 step protocol and annealing for 5 $\mathrm{s}$ at $60^{\circ} \mathrm{C}$. Relative gene expression was quantified by the use of the comparative cycle threshold $(\mathrm{Ct})$ method, normalized to EEF1A1 
Citation: Gunther K, Appelt-Menzel A, Kwok CK, Walles H, Metzger M, et al. (2016) Rapid Monolayer Neural Induction of induced Pluripotent Stem Cells Yields Stably Proliferating Neural Stem Cells. J Stem Cell Res Ther 6: 341. doi: 10.4172/2157-7633.1000341

and RPL6 expression. Fold difference calculation was done by $\Delta \Delta \mathrm{Ct}$ method.

\section{Imaging}

Phase contrast images were captured using DMIL LED and Leica Application Suite Software version 4.2.0 (both Leica Microsystems). For fluorescence microscopy Biorevo BZ-9000 (Keyence) and ECLIPSE Ti confocal laser scanning microscope (Nikon) and their respective software was utilized. Further image analysis was carried out with ImageJ.

\section{Results and Discussion}

\section{Initial validation of hiPSCs and neural induction}

Two previously published hiPSC-lines have been used in this study. The line IMR90 hiPSC clone 4 (IMR90-4, WiCell) was generated by Yu and colleagues [16] from female fetal lung fibroblasts by the application of lentiviral vectors (OCT4, SOX2, NANOG, LIN28). AR1034ZIMA hiPSC clone1 (ARIPS) [15] was obtained by reprogramming of male dermal fibroblasts from a skin punch biopsy (ethical report no: 96/11, University of Würzburg) using the polycistronic (OCT4, KLF4, SOX2, C-MYC) lentiviral vector STEMCCA [17]. Morphological analysis by phase contrast microscopy revealed typically formed hiPSC colonies, which were of homogeneous and compact shape (Figure 1A). We performed immunostainings to assess the expression of pluripotencyassociated markers such as nuclear OCT4 and SOX2, as well as cell surface antigen SSEA-4 (Figure 1B). Additionally, we assessed pluripotency by flow cytometry analysis by using antibodies directed against TRA-1-60. According to this analysis more than $98 \%$ of hiPSCs carried the pluripotency-associated marker TRA-1-60 (Figure 1C). Moreover, both cell lines display normal karyotype, are able to induce teratoma after injection in SCID mice and reveal a typical hiPSC gene expression profile as previously shown by us and Yu et al. $[15,16]$.

After comprehensive validation of the pluripotency status we initiated our two-step neural differentiation protocol (Figure 1D) by first applying the monolayer induction media to hiPSCs as previously published [14]. After the application of neural induction medium, cells first continued to form dense, roundly-shaped colonies but changed their morphology on day 4 as indicated by irregular appearing edges of the colonies and a heterogeneous morphology (Figure 2A). We observed further increase of heterogeneity and strong proliferation during the first days of monolayer cultivation. First passage was carried out at day 7 by splitting the cells and replating on fresh Matrigel -coated plates. After replating, cells changed their morphology drastically, losing the heterogeneity of partly compact colonies and loosely dispersed larger cells. Moreover, we observed changing to a relatively homogeneous culture consisting of highly proliferating cells exhibiting a neuroepithelial-like phenotype (Figure 2A). By that no further manual selection or purifying by sorting experiments seems to be required. Cells were grown under selective media conditions which promote neural induction until full confluency before passaging and further expanding until passage 5. To characterize differentiated cells at this stage, we investigated expression of several markers by immunostaining. According to this analysis, cells lost the expression of pluripotency marker OCT4 but remained in a strongly proliferative status as indicated by a high percentage of Ki67-positive cells (Figure 2B). Moreover, the differentiated cells homogeneously express NSC markers such as the cytoskeletal protein Nestin and transcription factors PAX6, SOX1 and SOX2 (Figures 2C and 2D). These data indicate that hiPSCs readily lost their pluripotent properties and adopted a multipotent, highly proliferative NSC status. To confirm this, we assessed the differentiation potential by applying unbiased
A

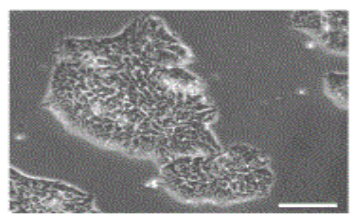

C

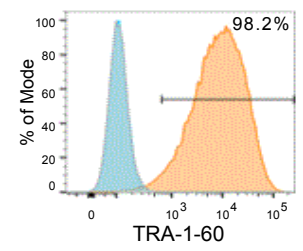

D

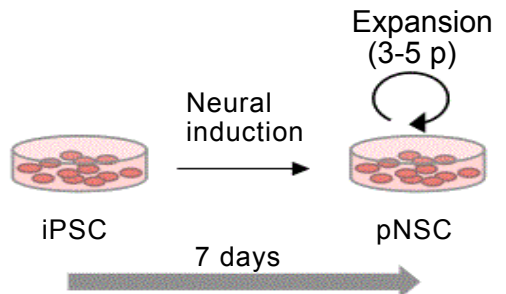

B
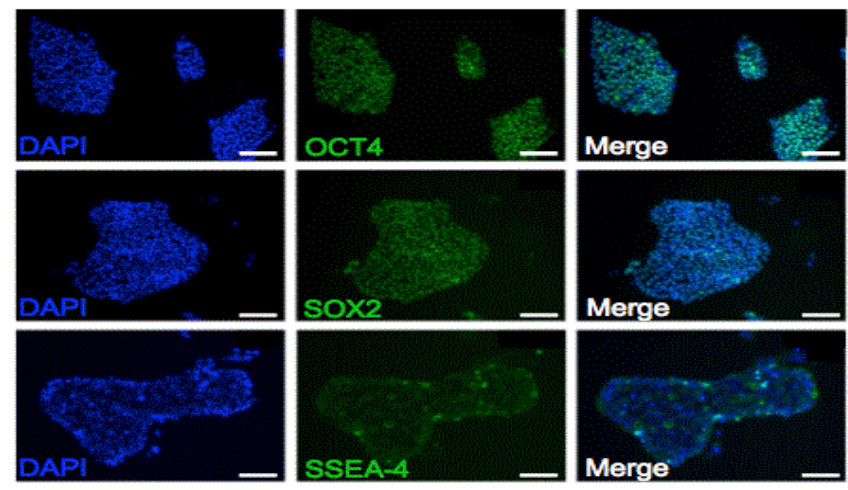

Expansion

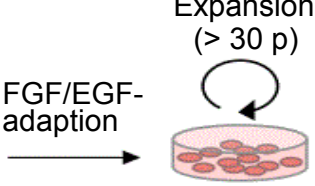

FGF/EGFNPC

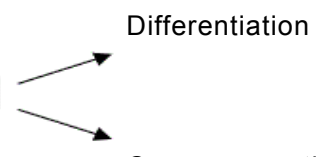

Cryopreservation

Figure 1: Characterization of hiPSCs and differentiation flowchart (A) Phase contrast image of iPS cells (ARiPS line) before neural induction shows compact, homogeneous morphology. (B) Representative immunocytochemical stainings against pluripotency-associated markers indicate homogeneous distribution of the nuclear proteins OCT4 and SOX2 as well as the cell surface antigen SSEA-4, nuclei are visualized using DAPI. (C) Flow cytometry analysis of pluripotency reveals $98.2 \%$ of iPSCs positive for the cell surface marker TRA-1-60. (D) Schematic presentation of the experimental workflow starting with differentiation of iPSCs into primitive NPCs (pNSC) within 7 days followed by expansion for 3-5 passages followed by adaptation to FGF/EGF-supplemented media. FGF/EGF-NPCs could be further expanded and differentiated into neural cell types as well as cryopreserved. Scale bars represent $100 \mu \mathrm{m}$. 


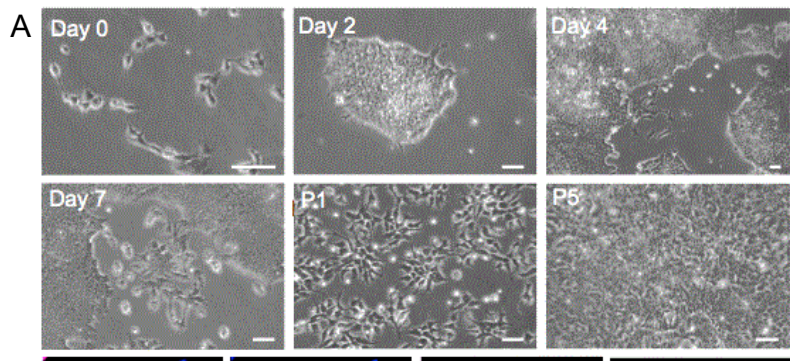

$\mathrm{B}$
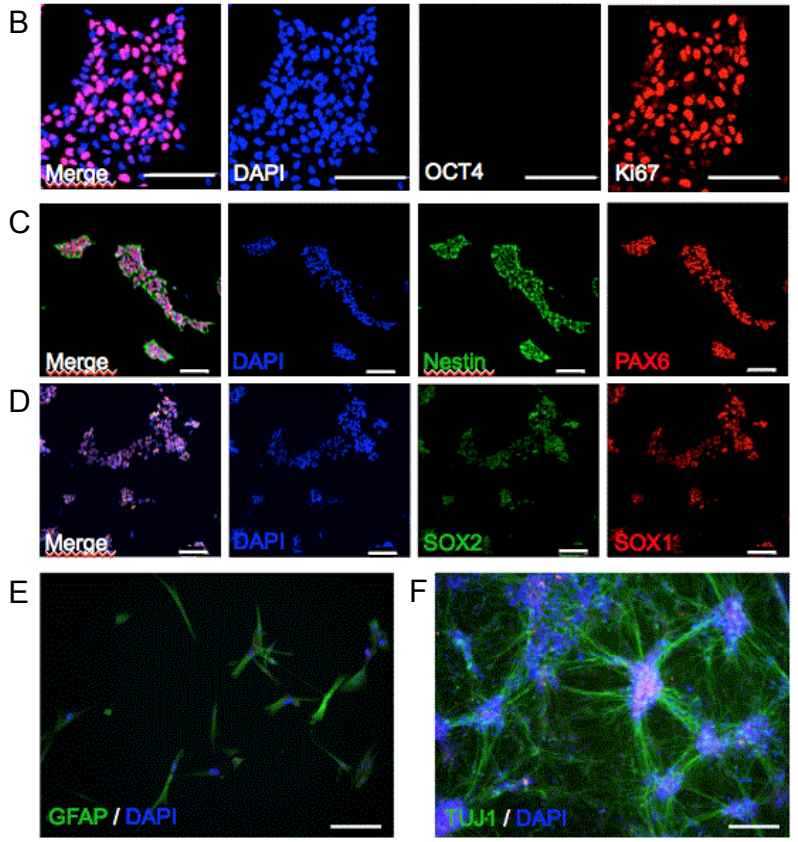

Figure 2: Derivation of pNSCs from hiPSCs (A) Phase contrast images from different time points of differentiation of iPSCs into primitive NSCs including day 0,2 , 4, 7 and passage 1 and 5 during expansion. (B) Imunocytochemical stainings showing the loss of the pluripotency protein OCT4 after differentiation but sustained proliferation indicated by positive nuclear Ki67 staining. Stainings for NSC proteins can be found in (C) and (D) represented by homogeneous distribution of Nestin and PAX6 (C) as well as SOX2 and SOX1 (D). Primitive NSCs could be differentiated in GFAP-positive astrocytes (E) and TUJ1-positive neurons (F). DAPI is used in all fluorescent images as nuclear staining. Scale bars are indicating $50 \mu \mathrm{m}$.

differentiation towards glial and neuronal lineages. We found high proportions of GFAP-positive cells as well as TUJ1-positive neurons in the differentiated cultures (Figures $2 \mathrm{E}$ and $2 \mathrm{~F}$ ). In conclusion, we could derive stably proliferating NSCs from hiPSCs within 7 days of monolayer culture that can be differentiated into neurons and astrocytes in vitro. Since these NSCs are maintained in commercially available media with partially undisclosed composition [14], we next decided to adapt them to more defined media conditions that are widely distributed in the neurobiological stem cell community.

\section{Adaptation of monolayer pNSCs to FGF/EGF medium conditions}

Koch et al. reported a pure population of long-term self-renewing rosette-type NSCs that are dependent on FGF and EGF which exhibit high clonogenicity and stable neurogenesis [8]. This NSC-type has been successfully used in numerous studies and can be derived from human pluripotent stem cells by neural induction and laborious manual isolation of neural rosettes. We wanted to investigate whether or not we can adapt the monolayer p-NSCs to this commonly used, more defined FGF/EGF-state. Therefore, we applied the FGF/EGFsupplemented media described by Koch et al. [8] to our monolayer derived pNSCs (Figure 1D). We counteracted the initially observed heterogeneity of the FGF/EGF-adapted cells (data not shown) by additionally supplementing low concentrations of CHIR99021 (0.8 $\mu \mathrm{M}$ ), a small molecule glycogen synthase kinase 3 (GSK3) inhibitor activating canonical Wnt signaling. CHIR99021 has been reported to induce sustained self-renewal of human NSCs [18,19] and particularly low concentrations appear to enhance homogeneity of neural progenitor populations [18]. Using this media we observed a prominent change in morphology from unstructured neuroepithelial islands to radially centered rosette-like clusters (Figure 3A). Those FGF/EGF-NPCs showed continuous proliferation and could be successfully expanded until passage 30 thus far while keeping their morphological features. Moreover, the cells kept their proliferation potential after cryopreservation. Next, we analyzed gene expression of relevant genes at different time points of differentiation by quantitative Real-Time PCR (Figure 3B and Table 1). We included samples from undifferentiated hiPSCs, monolayer-derived pNSCs (passage 1 and 5) and FGF/EGF-NPCs derived thereof. As expected the pluripotency marker gene POU5F1 is expressed only in hiPSCs, whereas SOX2 mRNA as detected in hiPSCs is slightly downregulated in pNSCs of passage 1 and 5 (0.4- and 0.5-fold, respectively), but shows almost equal expression in FGF/EGF-NPCs. Interestingly, NESTIN mRNA is found only at a basal level in early-passage pNSCs but strongly increased in NSCs from passage 5 (2.2-fold) and FGF/EGF-NPCs (7.32-fold). Even stronger upregulation was observed for the neural 
A
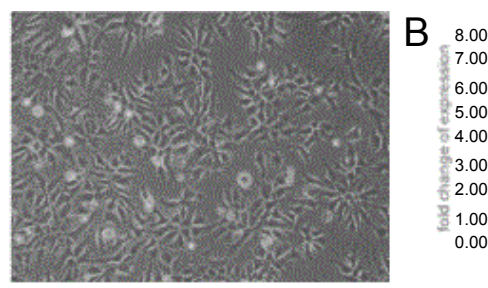

C

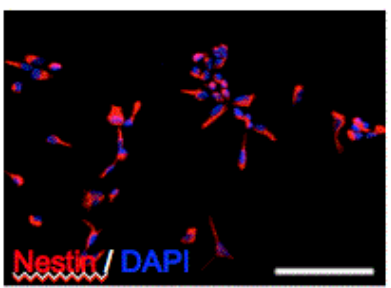

$\mathrm{D}$

F

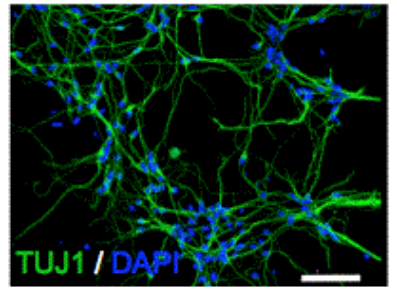

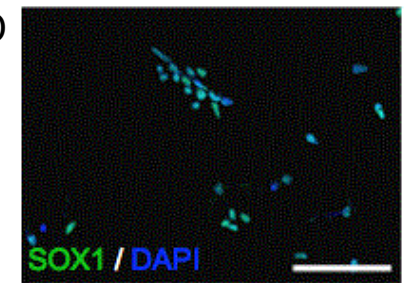
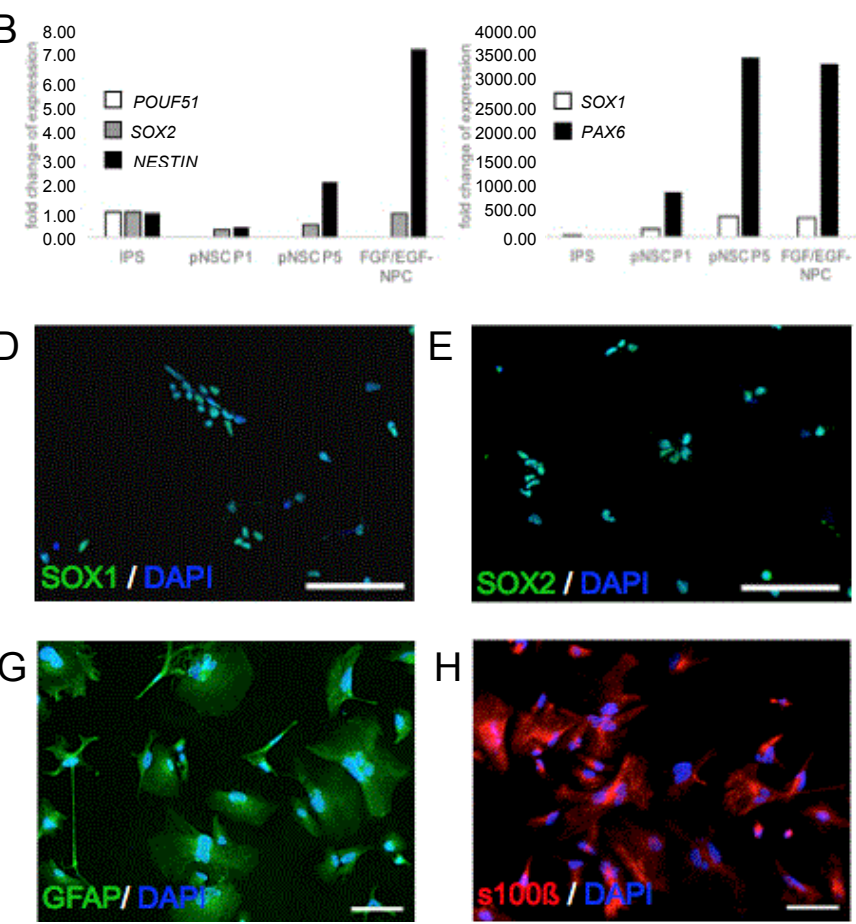

Figure 3: Characterization of FGF/EGF-dependent NPCs (A) A representative phase contrast image exhibiting rosette-like morphology in FGF/EGF-NPCs. (B) Gene expression analysis reveals downregulation of the pluripotency gene POU5F1 during differentiation and maintained expression of SOX2. Increased expression of Nestin was observed during differentiation into FGF/EGF-NPCs. The NSC marker genes SOX1 and PAX6 are strongly upregulated upon differentiation in primitive NSCs, showing even stronger increase after expanding for 5 passages and adapting to FGF/EGF-medium. (C) - (E) Immunocytochemical analysis confirms the presence of NSC marker proteins in FGF/EGF-NPCs. Differentiaton of FGF/EGF-NPCs yields in TUJ1-positive neurons (F) as well as GFAP- and S100ß-positive astrocytes ( $\mathrm{G}$ and $\mathrm{H}$, respectively). DAPI is used in all fluorescen stainings to counterstain nuclei. Scale bars are indicating $100 \mu \mathrm{m}$.

\begin{tabular}{|c|c|c|c|c|}
\hline & OCT4 & SOX2 & Nestin & SOX1 \\
\hline iPSCs & 1 & 1.0 & 1.0 & 1.0 \\
\hline pNSCs P1 & 0 & 0.4 & 0.4 & 1.0 \\
\hline pNSCs P5 & 0 & 0.5 & 2.2 & 893.4 \\
\hline FGF/EGF NPCs & 0 & 1.0 & 7.3 & 34.4 \\
\hline
\end{tabular}

Table 1: Relative fold-increase of gene expression.

marker genes SOX1 and PAX6. A major increase in SOX1 expression was revealed during the differentiation of hiPSCs to pNSCs (177.4-fold in passage 1 and 412.0-fold in passage 5) and expression remains high in FGF/EGF-NPCs (380.4-fold). Moreover, strong augmentation in PAX6 gene expression was observed during FGF-EGF-NPC derivation compared to hiPSCs (893.4-fold in P1 and 3494.6-fold in P5 of pNSCs, 3344.1-fold in FGF/EGF-NPCs). These data indicate that rapid change of morphology during both steps of differentiation correlates with the upregulation of neural markers at the molecular level. Furthermore, it suggests that the expansion of pNSCs up to 5 passages before changing the media conditions seems to be beneficial as revealed by gene expression changes between earlier and later passages.

\section{Characterization of FGF/EGF-NPCs}

To further characterize the obtained FGF/EGF-NPCs, immunocytochemical analysis was carried out, employing antibodies directed against various NSC markers. According to this analysis, FGF/ EGF-NPCs turned out to be positive for Nestin, PAX6, SOX1 and SOX2 confirming the NSC-profile of the cells (Figures 3C-3E). Next, we assessed the differentiation potential of FGF/EGF-NPCs by applying established directed differentiation protocols towards neurons and astrocytes. Neuronal differentiation resulted in a very high percentage of elongated cells with characteristic protrusions staining positive for the neuronal protein TUJ1 (Figure 3F). Moreover, we could observe efficient differentiation into astrocytes as judged by typical morphology as well as staining for glial cytoskeletal proteins such as GFAP and S100 $\beta$ (Figures $3 \mathrm{G}$ and $3 \mathrm{H}$ ). Taken together, these data demonstrate that FGF/EGF-dependent NPCs yielded neuronal cells and a high proportion of astrocytes after differentiation.

\section{Conclusion}

In summary, we present a two-step protocol that allows the rapid differentiation of hiPSCs into a FGF/EGF-dependent neural precursor population using a monolayer approach. This was achieved by the application of a previously published protocol to obtain pNSCs and a subsequent adaptation to defined media conditions containing bFGF, EGF and CHIR99021. Our procedure enables a relatively rapid and time saving derivation of highly proliferative FGF/EGF-NPCs with a characteristic neural marker profile. Further differentiation into neurons and astrocytes confirmed the stem cell characteristics. Thus, our protocol will help to obtain patient-specific neural cell types for biomedical applications such as disease modeling and cell replacement therapy. 
Citation: Gunther K, Appelt-Menzel A, Kwok CK, Walles H, Metzger M, et al. (2016) Rapid Monolayer Neural Induction of induced Pluripotent Stem Cells Yields Stably Proliferating Neural Stem Cells. J Stem Cell Res Ther 6: 341. doi: 10.4172/2157-7633.1000341

\section{Acknowledgments}

We would like to thank Heike Arthen and Alevtina Cubukova for excellent technical support. Additionally, we thank all members of the Stem Cell and Regenerative Group Würzburg and Dr. Ahmad Salti (University of Innsbruck, Austria) for helpful discussions and careful proofreading. This work was supported by grants from the Deutsche Forschungsgemeinschaft DFG, the European Union's Horizon 2020 research and innovation programme (ERA-NET E-Rare research programme on rare diseases) and by the FWF (Austrian Science Fund) Project Number FI030290.

\section{References}

1. Takahashi K, Tanabe K, Ohnuki M, Narita M, Ichisaka T, et al. (2007) Induction of pluripotent stem cells from adult human fibroblasts by defined factors. Cell 131: 861-872. [PubMed]

2. Yu DX, Marchetto MC, Gage HG (2013) Therapeutic translation of iPSCs for treating neurological disease. Cell Stem Cell 12: 678-688. [PubMed]

3. Marchetto MC, Carromeu C, Acab A, Yu D, Yeo GW, et al. (2010) A mode for neural development and treatment of Rett syndrome using human induced pluripotent stem cells. Cell 143: 527-539. [PubMed]

4. Brennand KJ, Simone A, Tran N, Gage FH (2012) Modeling psychiatric disorders at the cellular and network levels. Mol Psychiatry 17: 1239-1253. [PubMed]

5. Lippmann ES, Azarin SM, Kay JE, Nessler RA, Wilson HK, et al. (2012) Derivation of blood-brain barrier endothelial cells from human pluripotent stem cells. Nat. Biotechnol 30: 783-791. [PubMed]

6. Zhang SC, Wernig M, Duncan ID, Brüstle O, Thomson JA (2001) In vitro differentiation of transplantable neural precursors from human embryonic stem cells. Nat Biotechnol 19: 1129-1133. [PubMed]

7. Elkabetz Y, Panagiotakos G, A Shamy G, Socci ND, Tabar V, et al. (2008) Human ES cell-derived neural rosettes reveal a functionally distinct early neural stem cell stage. Genes Dev 22: 152-165. [PubMed]

8. Koch P, Opitz T, Steinbeck JA, Ladewig J, Brüstle O (2009) A rosette-type self-renewing human ES cell-derived neural stem cell with potential for in vitro instruction and synaptic integration. Proc Natl Acad Sci USA 106: 3225-3230. [PubMed]

9. Li W, Sun W, Zhang Y, Wei W, Ambasudhan R, et al. (2011) Rapid induction and long-term self-renewal of primitive neural precursors from human embryonic stem cells by small molecule inhibitors. Proc Natl Acad Sci USA 108: 8299-8304. [PubMed]

10. Reinhardt R, Glatza M, Hemmer K, Tsytsyura Y, Thiel TS, et al. (2013) Derivation and expansion using only small molecules of human neural progenitors for neurodegenerative disease modeling. PLoS One 8: e59252. [PubMed]

11. Kriegstein A, Buylla AA (2009) The glial nature of embryonic and adult neura stem cells. Annu Rev Neurosci 32: 149-184. [PubMed]

12. Gorris R, Fischer J, Erwes KL, Kesavan J, Peterson DA, et al. (2015) Pluripotent stem cell-derived radial glia-like cells as stable intermediate for efficient generation of human oligodendrocytes. Glia 63: 2152-2167. [PubMed]

13. Xia G, Santostefano K, Hamazaki T, Liu J, Subramony SH, et al. (2013) Generation of human-induced pluripotent stem cells to model spinocerebellar ataxia type 2 in vitro. J Mol Neurosci 51: 237-248. [PubMed]

14. Yan Y, Shin S, Jha BS, Liu Q, Sheng J, et al. (2013) Efficient and Rapid Derivation of Primitive Neural Stem Cells and Generation of Brain Subtype Neurons From Human Pluripotent Stem Cells Stem Cells Transl pp: 862-870. [PubMed]

15. Kadari A, Lu M, Li M, Sekaran T, Thummer RP, et al. (2014) Excision of vira reprogramming cassettes by Cre protein transduction enables rapid, robust and efficient derivation of transgene-free human induced pluripotent stem cells. Stem Cell Res 5: 47. [PubMed]

16. Yu J, Vodyanik MA, Otto KS, Bourget JA, Frane JL, et al. (2007) Induced pluripotent stem cell lines derived from human somatic cells. Science 318 1917-20. [PubMed]

17. Somers A, Jean JC, Sommer CA, Omari A, Ford CC, et al. (2010) Generation of transgene-free lung disease-specific human induced pluripotent stem cells using a single excisable lentiviral stem cell cassette. Stem Cells 28: 1728-1740. [PubMed]

18. Moya N, Cutts J, Gaasterland T, Willert K, Brafman DA (2014) Endogenous WNT signaling regulates hPSC-derived neural progenitor cell heterogeneity and specifies their regional identity. Stem cell reports 3: 1015-1028. [PubMed]

19. Bung R, Worsdorfer P, Thier MC, Lemke K, Gebhardt M, et al. (2016) Partial Dedifferentiation of Murine Radial Glia-Type Neural Stem Cells by Brn2 and c-Myc Yields Early Neuroepithelial Progenitors. Journal of Molecular Biology 1: 8.[PubMed] 